\title{
Bridging the Gap between Semantics and Multimedia Processing
}

\author{
Marcio Ferreira Moreno*, Guilherme Lima*, Rodrigo Santos*, Roberto Azevedo ${ }^{\dagger}$, and Markus Endler ${ }^{\ddagger}$ \\ *IBM Research, Brazil \\ mmoreno@br.ibm.com, guilherme.lima@ibm.com, rodrigo.costa@ibm.com \\ †EPFL, Switzerland \\ roberto.azevedo@epfl.ch \\ $\ddagger$ PUC-Rio, Brazil \\ endler@inf.puc-rio.br
}

\begin{abstract}
In this paper, we give an overview of the semantic gap problem in multimedia and discuss how machine learning and symbolic AI can be combined to narrow this gap. We describe the gap in terms of a classical architecture for multimedia processing and discuss a structured approach to bridge it. This approach combines machine learning (for mapping signals to objects) and symbolic AI (for linking objects to meanings). Our main goal is to raise awareness and discuss the challenges involved in this structured approach to multimedia understanding, especially in the view of the latest developments in machine learning and symbolic AI.
\end{abstract}

Keywords-Semantic Gap, Multimedia, Knowledge Representation, Semantic Web

\section{INTRODUCTION}

A classic problem in multimedia representation and understanding is the semantic gap problem [1]. It states that there is a big representational gap between the audiovisual signals that compose multimedia objects and the concepts represented by these signals. For instance, the dominant color and movement trajectory of a given set of pixels in a video clip, which are low-level characteristics of the clip, usually do not provide much information about the meaning of the set of pixels-at least not to computers. But recent developments in artificial intelligence (AI) are changing that.

Backed by large training datasets, current machine learning methods are able to extrapolate complex patterns from lowlevel multimedia data. These patterns are embodied in trained models which can be used to classify or identify persons and objects with reasonable speed and accuracy in images, audio clips, and to a lesser extent video clips [2].

But being able to identify persons and objects in multimedia data only solves half of the problem. To emulate human cognition and truly understand a scene-for instance, to determine who is doing what and the consequences of those actions-computers need additional information: they need common sense knowledge and domain knowledge, and also the capacity to infer new knowledge from preexisting knowledge. This is where symbolic AI comes in. The basic idea of symbolic $\mathrm{AI}$ is to describe the world, its entities, and their relationships using a formal language and to develop efficient algorithms to query and deduce things from these formal descriptions.
In this paper, we give an overview of the semantic gap problem in multimedia and discuss how machine learning (ML) and symbolic AI can be combined to narrow this gap. More specifically, we highlight the fact that what we call the semantic gap consists of many gaps which exist between the various layers of multimedia representation. A structured approach to tackle the gap as a whole is thus to tackle each of these smaller gaps individually, through a combination of ML (for mapping signals to objects) and symbolic AI (for linking objects to meaning).

Our main goal here is to raise awareness and discuss the challenges involved in this structured approach to multimedia understanding, especially in view of the latest developments in ML and symbolic AI.

The rest of the paper is organized as follows. In Section II, we define what we mean by "symbolic AI" and justify why we need it. In Section III, we describe how the semantic gap problem is distributed among the various layers of multimedia representation, and discuss a structured approach for multimedia understanding. In Section IV, we discuss the challenges involved in such a structured approach. Finally, in section $\mathrm{V}$, we draw our conclusions.

\section{Why We NeEd Symbolic AI}

To illustrate the kind of applications enabled by the combination of symbolic AI with machine learning and multimedia consider Figure 1.

Suppose we are given this picture and suppose the only thing we know about it is what we can infer from the image. We can see it depicts Jean-Paul Marat (assuming we can identify him), a stab wound, a blood-tainted knife, and a letter addressed to him and signed by Charlotte Corday (assuming we can read the contents of the letter). The analogy here is that we have extracted these information-or facts-using pattern matching. Although such basic facts allow us to perform simple computational tasks, such as keyword-based image classification and search, they are not enough to understand the image.

To truly understand what is being depicted in Figure 1 we need more than basic facts. We need (1) general knowledge about the world, (2) specific knowledge about the persons 


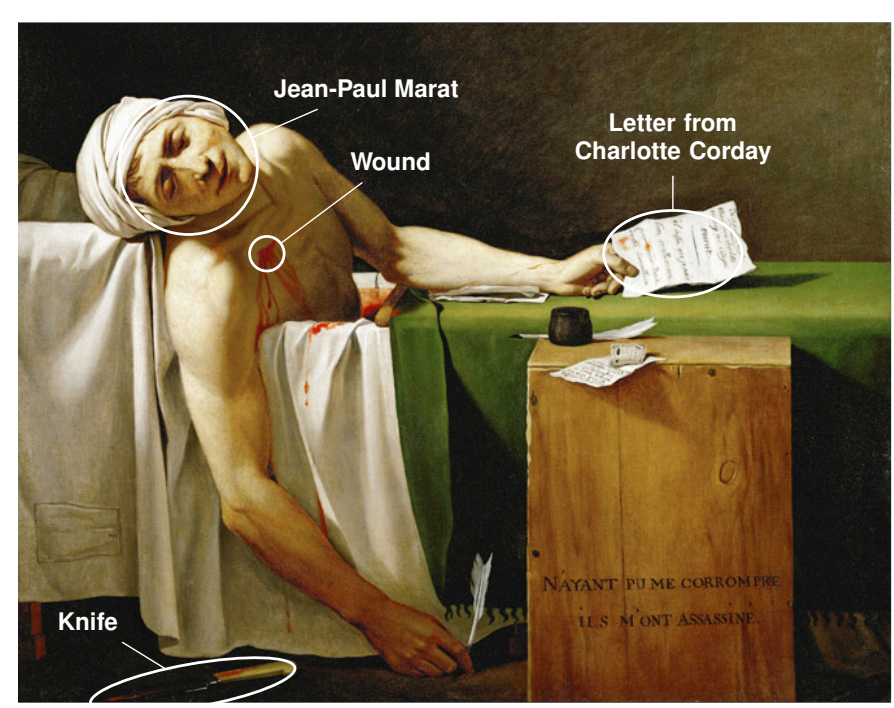

Fig. 1. The Death of Marat (detail), by Jacques-Louis David, 1793. (WikiMedia)

named, and (3) the capacity to combine general and specific knowledge with the facts extracted from the image in order to infer new facts.

Now suppose we are given (1), (2), and (3). From our general knowledge of the world, and possibly by further analyzing the image, we can assert with high confidence that Marat is holding the letter and that he has a stab wound on the chest. From this and from the blood-tainted knife depicted below him, we might infer that the depicted knife is the object that caused the wound. Since knifes are not autonomous beings, we might also conclude that someone (possibly himself) stabbed Marat in the chest. But who and why?

To answer these questions we will need more information. Suppose we are told that Marat was a journalist and political agitator, and one of the leaders of a radical political faction in the Reign of Terror period of the French Revolution (c. 1793). Suppose we are also told that Charlotte Corday, who signed the letter, was a declared political enemy of Marat-she blamed him for a number of killings in Paris and other cities and believed that he was a grave threat to the French Republic. Under the light of these new facts, we can conclude that Figure 1 looks like the scene of a political murder.

By combining this conclusion with the additional fact that Charlotte Corday is known to have murdered Jean-Paul Marat with a knife while he was in his bathtub, holding a letter from her, we can infer with a high degree of confidence that Figure 1 must be a graphical representation of this incident, that is, of the politically motivated assassination of Jean-Paul Marat by Charlotte Corday.

The derivation of this last fact from the visual patterns of Figure 1 has only been possible because we have had access not only to basic facts extracted from the image, but also to facts about the world (common sense knowledge) and about the depicted objects and persons (domain knowledge), and because we could combine all of these facts and make inferences.
One of the main goals of symbolic AI is to enable the representation and manipulation of pieces of knowledge by computers in ways that resemble or emulate the kind of manipulations performed by humans-manipulations similar to the considerations that enabled us to determine the true meaning of Figure 1. The combination of this capacity with multimedia opens up many possibilities. For instance, the Marat's murder example is an application of automated image understanding. Two related applications are video understanding and audio understanding, which are often more complex as they involve the extraction of temporal information.

Other applications of symbolic AI to multimedia include the semantic retrieval, classification, recommendation, and inspection of multimedia data-for example, to automatically identify suspicious activity in surveillance videos, generate age ratings for music and movies, and identify risk factors for diseases in medical images and videos.

\section{A Structured ApProach to Multimedia UNDERSTANDING}

\section{A. The many semantic gaps}

There is a hierarchy of layers of processing and representation separating raw multimedia data (arrays of bytes) from their semantics (meaning). The so-called semantic gap can occur between any two of these layers. To see why this is the case, consider Figure 2 which depicts the classic structure of a bottom-up pipeline for extracting semantics from multimedia content [3].

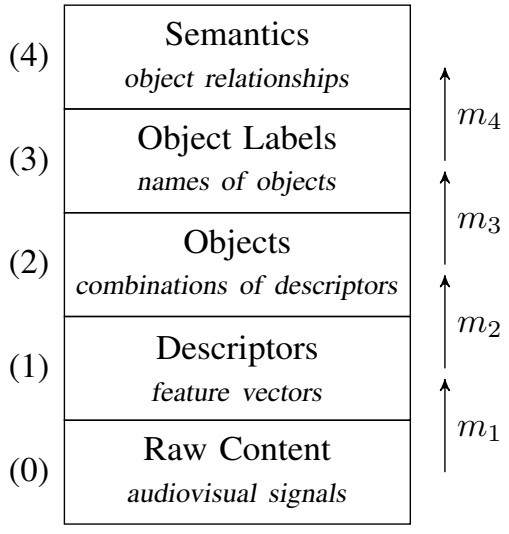

Fig. 2. Hierarchy of levels between raw content and its full semantics.

In the classical version of the pipeline, the first step, mapping $m_{1}$ in Figure 2, consists in extracting feature vectors describing low-level characteristics of the data, such as color histograms, pixel intensities, length of sounds, noise ratios, frame rates, etc. These feature vectors are then used by mapping $m_{2}$ as a basis for identifying objects.

Note that the gap is already present at mapping $m_{2}$. The question of how to extract or segment objects from feature vectors is far from simple, especially when only classical signal processing methods are considered. In the case of Figure 1, the mapping $m_{2}$ corresponds to the problem of grouping sets of features in order to identify contours and objects, for instance, 
the knife, the person, the letter, etc. (See [4]-[6] for a survey of approaches for tackling this problem for image, audio, and video data.)

Continuing upwards in the pipeline of Figure 2, once different objects are identified (layer 2), the next problem, mapping $m_{3}$, is to assign labels to these objects. In terms of Figure 1, this means to assign the labels "knife", "letter", "Marat", and "wound" to the appropriate segments of the image.

As before, mapping $m_{3}$ also has to deal with a gap. In this case, the gap between the objects identified by mapping $m_{2}$ and the labels which should represent them. The problem here is that an object might be mapped to multiple labels, the same label might refer to different objects, and the choice of label might depend on the context in which the object occurs (for instance, on its surroundings).

Most of the research related to the problem of the semantic gap in multimedia is concerned with mappings $m_{1}, m_{2}$ and $m_{3}$. That is, with bridging the distance between the raw content and the object labels. Even though this is crucial for multimedia understanding, as discussed in Section II, it comprises only half of the problem. After $m_{3}$, we are sill left with a gap between the labels and their meaning - a gap that must be tackled by mapping $m_{4}$. The idea is that after $m_{4}$, in layer 4 , we are no longer dealing with objects and labels, but with meaningful concepts. In case of Figure 1, a knife (the sharp object and its properties), Marat (the person and its history), etc.

We now shift the discussion to AI; to how the latest developments in machine learning and symbolic representation are contributing to narrow the gaps between the layers of Figure 2, and what are the challenges in these areas.

\section{B. Machine learning: From data to objects and labels}

In the last years, deep learning methods for visual object recognition and speech recognition have achieved unprecedented levels of accuracy, often surpassing by far that of traditional signal processing techniques [7], [8]. Given enough data and computational power, it is possible to train a neural network to effectively "solve" a specific pattern recognition task. The problem is that in the case of multimedia, the task is hardly specific and we are often dealing with data that is ambiguous or imprecise.

So, although deep learning has significantly improved our ability to map raw multimedia content to objects and labels (mappings $m_{1}, m_{2}$, and $m_{3}$ in Figure 2), there are still many open problems in this area. We discuss some of these in Section IV.

\section{Symbolic AI: From objects and labels to meanings}

The idea of symbolic AI is to describe the world, its entities, and their relationships using a formal language. And then to apply formal queries and reasoning over these descriptions. One can argue that the Semantic Web technologies [9] are the most successful realization of this idea, at least in terms of adoption. The use of ontologies (formal conceptualizations of particular domains) has become widespread, including within the multimedia community, where there is a growing interest in using ontologies to describe and derive higher-level facts from multimedia data [10]-[13]

Symbolic representation and reasoning is the key to bridge the gap between object labels and their meaning (mapping $m_{4}$ in Figure 2). Using an ontology language such as OWL [14] (a standard, Semantic Web technology), we can describe what the labels "knife" and "Marat" mean-what these entities are, how they behave, and what is their relation to the rest of the world. And from these descriptions, through formal reasoning, we can eventually derive the conclusion that the scene depicted in Figure 1 is that of a political murder.

That said, the semantic description of multimedia data is far from being a simple problem. The imprecise and ambiguous nature of multimedia requires descriptions that allows us to represent fuzziness and probability. Moreover, these same descriptions should allow us to speak of time and space, two fundamental dimensions of audiovisual data, and to link descriptions to the underlying fragments of data they describe. These and other limitations of current representation models have backed the development of hybrid models, such as Hyperknowledge [15], [16], which combines multimedia data and its semantic description under a single framework, and has support for spatial and temporal queries. We discuss these and other challenges next.

\section{Challenges}

There are still many challenges related to semantic gap in multimedia. We discuss some of these below.

1) Mulsemedia: Multiple sensorial media (Mulsemedia) is a recent trend that focuses on enriching traditional multimedia content with modalities that go beyond audio and video, that is, modalities such as haptic, olfactory, thermoceptic, etc. While there are many well-known algorithms for processing audiovisual signals, the algorithms and tools for processing mulsemedia are still in their infancy. Future research should tackle problems such as extracting meaning from sensorial media and aligning this meaning with the semantics extracted from traditional media contents [17], [18].

2) Moving objects: The detection and tracking of moving objects in videos is a well-known problem in video segmentation [19], [20]. Related to this problem and still a largely unexplored area of research, is the problem of describing such moving objects semantically in way that captures the notion that they have an identity even though they can possibly change through time.

For instance, when watching a video clip a person can easily determine that a woman walking on a sidewalk which gets temporarily occluded by a passing car is still the same woman after the car passes. Similarly, a child that puts on a mask is still the child, but now a masked one. The notion of time and change complicates not only the problem of identifying objects in audiovisual signals but also the problem of describing these objects and their changes-descriptions which might require a non-monotonic model of truth (things that are true now might not be true in the future). 
3) Real-time reasoning: Real-time reasoning over audiovisual streams is another challenging topic. In general, approaches based on deep learning introduce non-negligible delays that make them unsuitable for applications that require near realtime performance. Things are even worse on the symbolic side, where the complexity of reasoning tasks is often related to the size of the knowledge base. Real-time reasoning over multimedia data requires processing and correlating spatial (intra-frames) and temporal (inter-frames) data with corresponding symbolic descriptions on the fly. This is pretty much an open problem which has to be investigated by the community.

\section{Final Remarks}

In this paper, we discussed the problem of the semantic gap in multimedia representation in the view of the latest developments in AI. We described this gap in terms of a classical architecture for multimedia processing and discussed how the gap is distributed among the layers of this architecture. We noted that a promising approach for bridging this gap is to do so in a structured manner through a combination of machine learning (for mapping signals to objects) and symbolic AI (for linking objects to meanings). We discussed the current challenges involved in this approach and also future challenges, which will require further advances in IA methods and technologies.

\section{REFERENCES}

[1] L. F. Sikos, Description Logics in Multimedia Reasoning. Springer, 2017.

[2] W. Zhang, T. Yao, S. Zhu, and A. E. Saddik, "Deep learning-based multimedia analytics: A review," ACM Trans. Multimedia Comput. Commun. Appl., vol. 15, no. 1s, pp. 2:1-2:26, January 2019.

[3] J. S. Hare, P. H. Lewis, P. G. B. Enser, and C. J. Sandom, "Mind the gap: Another look at the problem of the semantic gap in image retrieval," in Electronic Imaging, 2006.

[4] D. Brezeale and D. J. Cook, "Automatic video classification: A survey of the literature," IEEE Transactions on Systems, Man, and Cybernetics, Part C (Applications and Reviews), vol. 38, no. 3, pp. 416-430, May 2008.
[5] Z. Fu, G. Lu, K. M. Ting, and D. Zhang, "A survey of audio-based music classification and annotation," IEEE Transactions on Multimedia, vol. 13, no. 2, pp. 303-319, April 2011.

[6] W. Khan, "A survey: Image segmentation techniques," International Journal of Future Computer and Communication, vol. 3, pp. 89-93, 2014.

[7] P. N. Druzhkov and V. D. Kustikova, "A survey of deep learning methods and software tools for image classification and object detection," Pattern Recognition and Image Analysis, vol. 26, no. 1, pp. 9-15, 2016.

[8] F. Alías, J. C. Carrié, and X. Sevillano, "A review of physical and perceptual feature extraction techniques for speech, music and environmental sounds," Applied Sciences, vol. 6, p. 143, 052016.

[9] P. Hitzler, M. Krötzsch, and S. Rudolph, Foundations of Semantic Web Technologies. Chapman \& Hall, 2010.

[10] R. Nevatia, J. Hobbs, and B. Bolles, "An ontology for video event representation," in 2004 Conference on Computer Vision and Pattern Recognition Workshop, June 2004, pp. 119-119.

[11] C. Saathoff and A. Scherp, "Unlocking the semantics of multimedia presentations in the web with the multimedia metadata ontology," in Proc. 19th International Conference on World Wide Web, ser. WWW '10. ACM, 2010, pp. 831-840.

[12] S. Chaudhury, A. Mallik, and H. Ghosh, Multimedia Ontology: Representation and Applications. CRC Press, 2015.

[13] A. Carbonaro and R. Reda, "Semantic content representation to meet the challenges of video analysis in multimedia entertainment," in 2018 IEEE 29th Annual International Symposium on Personal, Indoor and Mobile Radio Communications (PIMRC), 2018, pp. 42-47.

[14] W3C OWL WG, "OWL 2 Web Ontology Language document overview (second edition)," W3C, Recommendation, December 2012, http://www. w3.org/TR/2012/REC-owl2-overview-20121211/.

[15] M. F. Moreno, R. Brandao, and R. Cerqueira, "Extending hypermedia conceptual models to support hyperknowledge specifications," International Journal of Semantic Computing, vol. 11, no. 01, pp. 43-64, 2017.

[16] _ "NCM 3.1: A conceptual model for hyperknowledge document engineering," in Proceedings of the 2016 ACM Symposium on Document Engineering, ser. DocEng '16. New York, NY, USA: ACM, 2016, pp. $55-58$.

[17] E. a. B. Saleme, C. A. S. Santos, R. A. Falbo, G. Ghinea, and F. Andres, "Towards a reference ontology on mulsemedia systems," in Proceedings of the 10th International Conference on Management of Digital EcoSystems, ser. MEDES '18. New York, NY, USA: ACM, 2018, pp. 23-30.

[18] T. Naravane and M. Lange, "Organoleptic and sensory ontology," in JOWO, 2017.

[19] A. Yilmaz, O. Javed, and M. Shah, "Object tracking: A survey," ACM Comput. Surv., vol. 38, no. 4, 2006.

[20] G. Ciaparrone, F. L. Sánchez, S. Tabik, L. Troiano, R. Tagliaferri, and F. Herrera, "Deep learning in video multi-object tracking: A survey," 2019. 